\title{
Simplex underwater acoustic communications using passive time reversal
}

\author{
Lin Sun ${ }^{1,2}$, Ruo $\mathrm{Li}^{1,2}$, Bo Zou ${ }^{1,2}$, Zhaoqi Dong ${ }^{1,2}$, Haisen $\mathrm{Li}^{1,2, \mathrm{e}}$ \\ ${ }^{1}$ Acoustic Science and Technology Laboratory, Harbin Engineering University, Harbin 150001, \\ China; \\ ${ }^{2}$ College of Underwater Acoustic Engineering, Harbin Engineering University, Harbin 150001, China; \\ ehsenli@126.com
}

Keywords: passive time reversal; underwater acoustic communications; simplex; adaptive linear equalization

\begin{abstract}
The spatial-temporal compression achieved by time reversal (TR) can reduce the inter-symbol interference (ISI) and increase the signal strength with low computation complexion. The active TR needs two-way propagation to implement focusing, so it cannot be used in simplex underwater acoustic communications. Based on the one-way propagation and spatial-temporal focusing property of passive TR, a simplex underwater acoustic communication scheme using passive TR is presented. The presented scheme is considered in two scenarios: uplink transmission from a single send-only element to an array and downlink transmission from an array to a single receive-only element, a multi-channel linear equalizer (LE) is adopted for uplink transmission and a single channel LE is used for downlink transmission to remove residual interference. Theory analysis shows that ideally the presented scheme can minimize the ISI and noise. Experiment results show that comparing with the performance of simplex communication scheme using passive TR alone and simplex communication scheme using a LE alone, the performance of presented scheme is the best.
\end{abstract}

\section{Introduction}

In recent years, time reversal (TR) has attracted increasing attention in underwater acoustic communications due to its simple hardware structure and low computation complexity. TR can be divided into active $\mathrm{TR}^{[1]}$ and passive $\mathrm{TR}^{[2-7]}$. The focusing of active $\mathrm{TR}$ is achieved through two-way propagation in real underwater acoustic channel. Passive TR only needs one-way propagation to implement numerical spatial-temporal focusing, so it can be used in simplex acoustic communications. Simplex acoustic communications include two scenarios: uplink transmission from a send-only single element to an array and downlink transmission from an array to a receive-only single element. The passive TR communication scheme proposed by Song et al. ${ }^{[2]}$ and Zhang et al. ${ }^{[6]}$ can be used in simplex uplink transmission, but when the bit error rate (BER) is relatively high, the decision feedback equalizer (DFE) in the scheme can cause error propagation which will decrease communication performance. The passive TR communication scheme proposed by Sun $e t$ al $^{[7]}$ can be used in simplex downlink communications, but the scheme has been only analyzed in theory and has not proposed the method to eliminate the residual interference. In order to overcome the shortcoming of [2], [6] and [7], a simplex underwater acoustic communication scheme using passive TR is presented. The presented scheme is considered in two scenarios: uplink transmission and downlink transmission. For uplink transmission, a multi-channel linear equalizer (LE) adopted in the presented scheme not only can fully exploit space diversity to suppress residual interference but also can avoid error propagation. For downlink transmission of presented scheme, a single channel LE is used to eliminate the residual interference without error propagation. 


\section{Theory analysis}

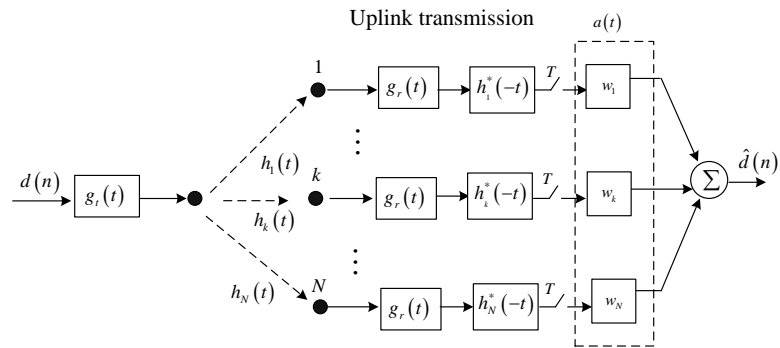

(a)

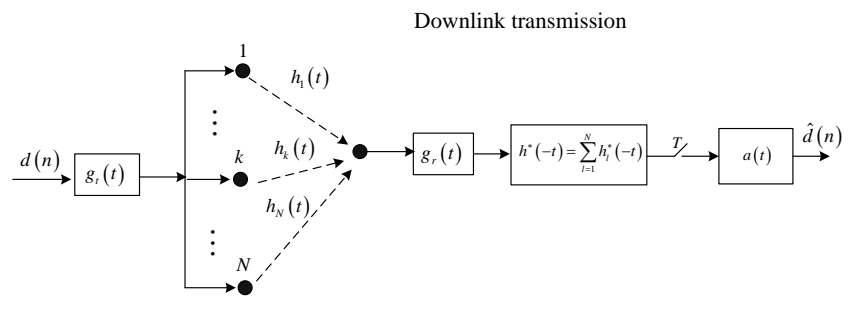

(b)

Fig. 1. The system model of presented scheme for two scenarios.

With reference to Fig .1, the signal after passive TR processing can be denoted by

$$
y(t)=\sum_{n} d(n) f(t-n T)+z(t)
$$

Where $d(n)$ denotes the sequence of data symbols which is transmitted at a symbol rate $1 / T, z(t)$ is the noise after passive TR processing, $f(t)$ is the composite channel response.

$$
\begin{aligned}
& f(t)= \begin{cases}\sum_{k=1}^{N} g_{t}(t) * g_{r}(t) * h_{k}(t) * h_{k}^{*}(-t) & \text { uplink } \\
\sum_{k=1}^{N} g_{t}(t) * g_{r}(t) * h_{k}(t) * h_{k}^{*}(-t)+\sum_{k=1}^{N} \sum_{l=1, l \neq k}^{N} g_{t}(t) * g_{r}(t) * h_{k}(t) * h_{l}^{*}(-t) \text { downlink }\end{cases} \\
& z(t)= \begin{cases}\sum_{k=1}^{N} n_{k}(t) * g_{r}(t) * h_{k}^{*}(-t) & \text { uplink } \\
\sum_{k=1}^{N} n(t) * g_{r}(t) * h_{k}^{*}(-t) & \text { downlink }\end{cases}
\end{aligned}
$$

In Eq. 2 and Eq. 3, $h_{k}(t), k=1, \cdots, N$ denotes the channel response between the $k$ th element of array and the single element, $g_{t}(t)$ and $g_{t}(t)$ denote the transmit filter and receive filter respectively, $n_{k}(t), k=1, \cdots, N$ is the noise received by the $k$ th element of the array for uplink transmission, and $n(t)$ is the channel noise received by the receive-only single element for downlink transmission. We can see from Eq.2 that the $f(t)$ for downlink transmission is additional affected by the cross-correlation of channel responses, so the spatial diversity performance of downlink transmission is inferior to that of uplink transmission.

After sampling $y(t)$ at the symbol rate $1 / T$, we can get

$$
y(m T)=d(m) f(0)+\sum_{n \neq 0} d(m-n) f(n T)+z(m T)
$$

If the transmitted energy per symbol is set to $P$, the data symbols are uncorrelated, the channel noises are uncorrelated and the power spectral density of noise is $N_{0}$, then the SINR at the output of passive TR can be given by 


$$
\operatorname{SINR}_{t r}=\left[\left(\operatorname{SIR}_{t r}\right)^{-1}+\left(S N R_{t r}\right)^{-1}\right]^{-1}=E\left[|d(m) f(0)|^{2}\right] / E\left[\left|\sum_{n \neq 0} d(m-n) f(n T)+z(m T)\right|^{2}\right]
$$

Where $S I R_{t r}=|f(0)|^{2} / \sum_{n \neq 0}|f(n T)|^{2}$ and $S N R_{t r}=P \cdot|f(0)| / N_{0}$. From Eq. 5, we can see that there are residual ISI after the passive TR processing. Combining Eq. 5 with Eq. 2, we can further conclude that the $S I R_{t r}$ for downlink transmission is lower than that for uplink transmission due to cross-correlation of channel responses.

In order to eliminate residual interference, a LE is used in the presented scheme. From Fig.1, we can see that $a(t)$ is a multi-channel equalizer for uplink transmission and $a(t)$ is a single channel equalizer for downlink transmission. If the taps of $a(t)$ were infinite, the output SINR should be ${ }^{[4]}$

$$
\operatorname{SINR}_{e q}=\left[\int_{-1 / 2 T}^{-1 / 2 T} \frac{T}{1+\frac{\left(P / N_{0}\right)}{N \int_{-\infty}^{+\infty} G_{t}^{2}(f) d f} F[f]} d f\right]^{-1}-1
$$

$G_{t}(f)$ denotes the spectrum of $g_{t}(t), F[f]$ is the folded spectrum of $F(f)$. From Eq. 6, we notice that the $\operatorname{SINR}_{e q}$ for uplink transmission is same as that for downlink transmission on ideal condition, and residual ISI could be completely eliminated when $P / N_{0} \rightarrow \infty$.

\section{Experiment}

The experiments were carried out in a channel pool. The array has two elements. The first element was placed at $1.8 \mathrm{~m}$ depth, and the second element was deployed at $2 \mathrm{~m}$. The single element was placed at $2 \mathrm{~m}$ depth. The communication distance was $6.5 \mathrm{~m}$. The probe signal was a $50 \mathrm{~ms} 8-16 \mathrm{kHz}$ chirp with a Hamming window. The carrier signal was the same as the probe signal. The number of data symbols was 5100, 200 symbols ahead were used as the training symbol, the symbol rate was $2400 \mathrm{symbol} / \mathrm{s}$, and binary phase-shift keying (BPSK) was used to encode the data symbols. The taps of equalizer were 14; that is to say, each filter of the multi-channel equalizer for uplink transmission had 7 taps. The adopted adaptive algorithm was RLS algorithm, and the forgetting factor was set to 0.999 .

Fig. 2 displays the performance of three communication scheme when the input SNR is 24dB: (1) the presented communication scheme using passive TR and linear equalization; (2) the simplex communication scheme using passive TR alone; (3) the simplex communication scheme using a LE alone. From Fig. 2 we can see, the performance of presented scheme is the best among three communication scheme. Moreover, it is also worth notice that the BER in Fig. 2 (b) is 1\% lower than Fig. 2 (e), the reason for this is that the composite channel response for uplink transmission has less side-lobe than downlink transmission. Due to the BER after passive TR for uplink transmission is relatively low, the scatter plot of present scheme for uplink transmission is superior to that for downlink transmission when the BER after equalization is same. 


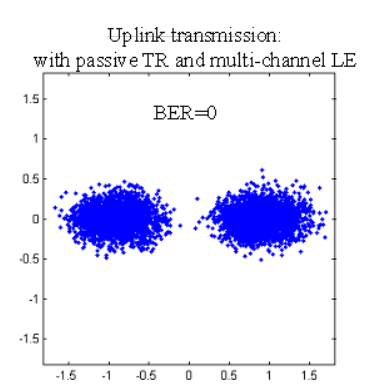

(a)

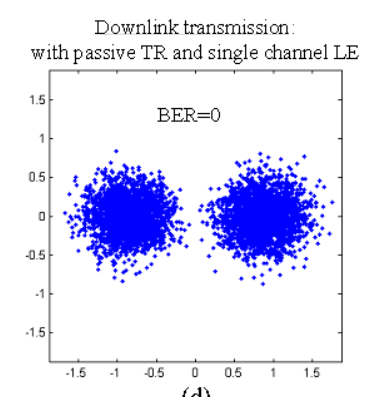

(d)

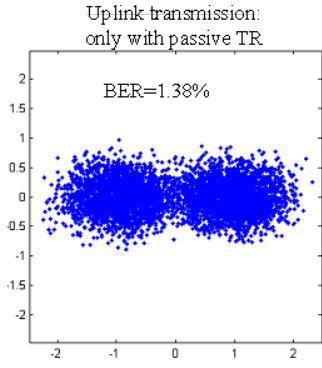

(b)

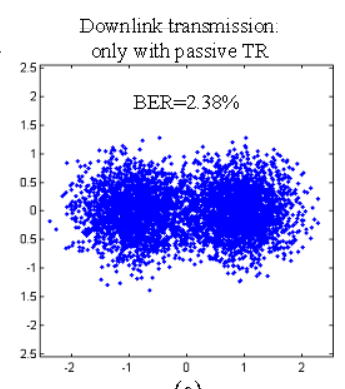

(e)

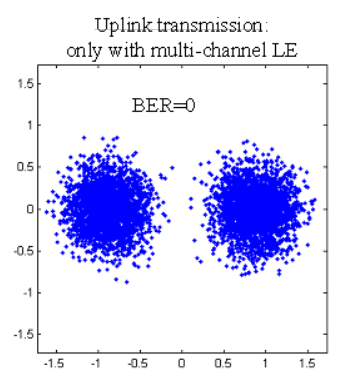

(c)

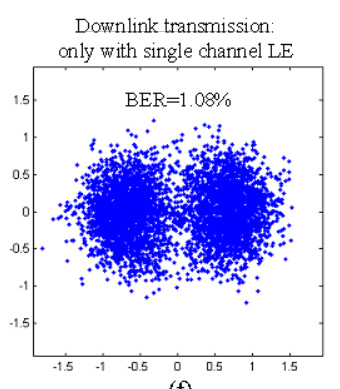

(f)

Fig. 2. Comparing the performance of three communication schemes for two scenarios

\section{Summary}

A simplex underwater acoustic communication scheme using passive TR and a LE is presented in this paper. The principle of presented scheme is analyzed in theory and the performance of presented scheme is verified through experiments. Results demonstrate that comparing with the performance of simplex communication scheme using passive TR alone and simplex communication scheme using a $\mathrm{LE}$ alone, the performance of presented scheme is the best.

\section{Acknowledgment}

The work was financially supported by the Specialized Research Fund for the Doctoral Program of Higher Education of China (Grant No. 20112304130003).

\section{References}

[1] G. F. Edelmann, T. Akal, W. S. Hodgkiss, S. Kim, W. A. Kuperman, and H. C. Song: Ocean. Eng. Vol. 27 (2002), p. 602

[2] H. C. Song, P. Roux, W. S. Hodgkiss, W. A. Kuperman, T. Akal, and M. Stevenson: Ocean. Eng. Vol. 31(2006), p. 170.

[3] H. C. Song, Kim, W. S. Hodgkiss, W. A. Kuperman, W. A., and Stevenson, M.: Acoust. Soc. Am. Vol. 128 (2010), p. 2920.

[4] S. E. Cho, H. C. Song and W. S. Hodgkiss: Acoust. Soc. Am. Vol. 132(2012), p. 5.

[5] A. Song and M. Badiey: Acoust. Soc. Am. Vol. 131(2012), p. 281.

[6] G. S. Zhang, and H. F. Dong: Applied Acoustics, Vol. 73(2012), p. 433.

[7] L. Sun, R. Li, and T. Zhou: Journal of Harbin Engineering University, Vol. 10 (2013): p. 312. 